\section{Does UKLS strategy increase the yield of screen-detected lung cancers? A comparison with ITALUNG}

The optimal strategy to increase the benefit of low dose computed tomograph (LDCT) screening of lung cancer in terms of as high as possible number of discovered cancers and to reduce the costs, in terms of as low as possible number of LDCT examinations and of interventions on benign lesions, is not established.

Field and co-workers recently reported $^{1}$ the results of lung cancer screening with LDCT in the UKLS RCT pilot study that selected eligible subjects with a validated individual risk prediction model, invited potential candidates by mail and applied the Wald Single Screen Design $^{2}$ with nodule management based on volumetry. ${ }^{1}$

We compared (table 1) some data of UKLS RCT pilot study with those of ITALUNG RCT that selected eligible subjects based on age and smoking history, invited potential candidates by mail and involved four annual LDCT screening rounds with nodule management based on diameter measurement. ${ }^{34}$

In the UKLS pilot study, higher rates of screen-detected primary lung cancers
( $2.1 \%$ vs $1.7 \%)$ and of stage I-II lesions ( $86 \%$ vs $68 \%$ ) were observed. These features may be accounted for different population's characteristics including older age (mean 67 vs 61 years), higher male/female ratio (3.01 vs 1.79 ), higher frequency of asbestos exposure $(36.0 \%$ vs $6.6 \%)$, higher prevalence of respiratory disease $(52.1 \%$ vs $35.1 \%$ ) and familial history for lung cancer $(24.6 \%$ vs $16.8 \%)$ in UKLS screens. In particular, prevalence of lung cancer at LDCT screening can be as high as $4.2 \%$ in subjects exposed to asbestos. ${ }^{5}$ On the other hand, the majority of the screens in UKLS were former smokers, whereas they were current smokers in ITALUNG. Twelve-month LDCT were obtained in

Table 1 Screens risk profile and results of LDCT in pilot UKLS and ITALUNG RCT

\begin{tabular}{|c|c|c|}
\hline & Pilot UKLS & ITALUNG \\
\hline Age (years) of selected subjects & $50-75$ & $55-69$ \\
\hline Eligibility criteria & $\begin{array}{l}\text { 5-year lung cancer risk of } \geq 5 \% \text {, based on the } \\
\text { Liverpool Lung Project } 22 \text { risk prediction model }\end{array}$ & Smokers or former smokers of $\geq 20$ pack/years \\
\hline \multicolumn{3}{|l|}{ Sample size } \\
\hline Control arm & 2027 & 1593 \\
\hline Screened arm & 2028 & 1613 \\
\hline \multicolumn{3}{|l|}{ Screeens' characteristics } \\
\hline Mean age at randomisation (years SD) & $67(4.1)$ & $61(4.2)$ \\
\hline Gender (male/female ratio) & $1529 / 499(3.06)$ & $1035 / 578(1.79)$ \\
\hline Current smokers & $777(38.3 \%)$ & $1060(65.7 \%)$ \\
\hline Ex-smokers & $1249(61.6 \%)$ & $553(34.3 \%)$ \\
\hline Never smokers & $2(0.1 \%)$ & 0 \\
\hline \multicolumn{3}{|l|}{ Smoking duration } \\
\hline 10-19 years* & $117(5.8 \%)$ & $1(0.06 \%)$ \\
\hline $20+$ years* & $1895(93.4 \%)$ & $1612(99.94 \%)$ \\
\hline Unknown* & $14(0.7 \%)$ & 0 \\
\hline$\%$ Asbestos exposed & $763(36 \%)$ & $93(6.6 \%) \dagger$ \\
\hline$\%$ With history of respiratory disease $\neq$ & $1056(52.1 \%)$ & $494(35.1 \%) \dagger$ \\
\hline$\%$ With history of blood cancer§ & $26(1.28 \%)$ & Not eligible \\
\hline$\%$ With history of solid tumourq & $378(18.6 \%)$ & Not eligible \\
\hline$\%$ With family history of lung cancer & $498(24.6 \%)$ & $237(16.8 \%) \dagger$ \\
\hline$\%$ With family history of other cancer (not lung)** & $1026(50.6 \%)$ & $640(45.5 \%) \dagger$ \\
\hline Baseline LDCT completed & $1994(98.3 \%)$ & $1406(87.2 \%)$ \\
\hline LDCT detected primary lung cancers & $42 / 1994(2.1 \%)$ & $25 / 1406(1.7 \%)+\dagger$ \\
\hline At baseline scan & $34 / 1994(1.7 \%)$ & $21 / 1406(1.4 \%)$ \\
\hline Adenocarcinoma & $25 / 42(59.5 \%)$ & $13 / 25(52.0 \%)$ \\
\hline Stage I lung cancer & $28 / 42(66.7 \%)$ & $14 / 25(56.0 \%)$ \\
\hline Stage I or II lung cancer & $36 / 42(85.7 \%)$ & $17 / 25(68.0 \%)$ \\
\hline Surgical resection & $35 / 42(83.3 \%)$ & $17 / 25(68.0 \%)$ \\
\hline Subjects undergoing 12-month scan LDCT & 1015/1994 (50.9\%)‡‡ & $1356(96.4 \%)$ \\
\hline Overall category 3 and 4 nodules $\S \S$ & $536 / 1994(26.8 \%)$ & $426 / 1406(30.2 \%)$ \\
\hline Of these, subjects found to have lung cancer & $42 / 536(7.8 \%)$ & $25 / 426(5.8 \%)$ \\
\hline Surgical resection for benign disease & $4 / 39(10.3 \%)$ & 1/21 (4.7\%)ๆๆ \\
\hline
\end{tabular}

\footnotetext{
*All smoking (cigarettes, cigars, pipes) duration figures refer to current and ex-smokers combined.

tInformation available in 1406 subjects undergoing baseline LDCT.

¥Asthma, bronchitis, TB, pneumonia, COPD or emphysema.

$\S$ Leukaemia or lymphoma, including Hodgkin's.

१Cancers of brain, head and neck, oesophagus, breast, colon or 'other'.

t+Data of ITALUNG refer to baseline and first annual repeat LDCT screening rounds.

¥¥Due to evidence at baseline of nodules $>3 \mathrm{~mm}$ diameter.

${ }^{* *}$ Cancers of brain, head and neck, oesophagus, breast, colon or 'other'.

§§Category 3 nodules correspond to: solid nodules with 5-9.9 mm diameter; part-solid nodules with non-solid component $>5 \mathrm{~mm}$ diameter and solid component of 3-9.9 mm diameter; non-solid nodules $\geq 5 \mathrm{~mm}$ diameter.

Category 4 nodules correspond to: solid nodules $\geq 10 \mathrm{~mm}$ diameter; part-solid nodules with solid component $\geq 10 \mathrm{~mm}$ diameter.

१ๆ One case of atypical adenomatous hyperplasia reclassified as adenocarcinoma in 2015.
} 
$50.9 \%$ of UKLS and $96.4 \%$ of ITALUNG screens. However, the rate of intervention for benign lesions was lower in ITALUNG (4.7\% vs $10.3 \%)$.

In conclusion, the UKLS recruitment strategy increases the yield of screendetected lung cancer compared with ITALUNG, but the gain is mild (19\%). This supports the view that incorporation of additional risk factors of lung cancers as pulmonary emphysema ${ }^{6}$ and serum biomarkers ${ }^{7}$ in selection criteria of eligible subjects for LDCT screening might increase such a yield. Moreover, above data demonstrate feasibility of comparison of risk factors in subjects recruited in RCTs of lung cancer screening that is required before pooling and joint analysis of lung cancer mortality data. ${ }^{9}$

Mario Mascalchi, ${ }^{1}$ Andrea Lopes Pegna, ${ }^{2}$ Laura Carrozzi, ${ }^{3}$ Francesca Carozzi, ${ }^{4}$ Fabio Falaschi, ${ }^{5}$ Giulia Picozzi, ${ }^{6}$ Eugenio Paci ${ }^{7}$

${ }^{1}$ Department of Experimental and Clinical Biomedical Sciences, University of Florence, Florence, Italy ${ }^{2}$ Pneumonology Department, Careggi Hospital, Florence, Italy

${ }^{3}$ Cardiopulmonary Department, University Hospital, Pisa, Italy

${ }^{4}$ Analytical Cytology and Bio-Molecular Unit, Institute for Oncological Study and Prevention, Florence, Italy ${ }^{5}$ 2nd Radiology Department, University Hospital of Pisa, Pisa, Italy

${ }^{6}$ Radiodiagnostic Unit, Institute for Oncological Study and Prevention, Florence, Italy

${ }^{7}$ Department of Epidemiology, Institute for Oncological Study and Prevention, Florence, Italy

Correspondence to Professor Mario Mascalchi, "Mario Serio" Department of Experimental and Clinical Biomedical Sciences, University of Florence, Viale Pieraccini, Florence 50133, Italy; m.mascalchi@dfc. unifi.it

Contributors MM devised the paper and wrote the paper draft, ALP reviewed the recruitment data and critically discussed the paper. LC critically discussed the risk factors data. $F C$ critically reviewed the manuscript. FF critically discussed the radiological data. GP reviewed radiological data. EP critically revised the paper.

Competing interests None declared.

Ethics approval Local Ethic Committees of Florence, Pistoia and Pisa Hospitals (approval number 29-30 of 30 September 2003; number 23 of 27 October 2003; and number 00028543 of 13 May 2004.

Provenance and peer review Not commissioned; externally peer reviewed.

Data sharing statement The authors intend to share the data from the ITALUNG study concerning recruitment, risk factors of eligible subjects and results of LDCT screening.

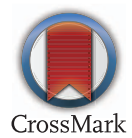

To cite Mascalchi M, Lopes Pegna A, Carrozzi L, et al. Thorax 2016;71:950-951.

Received 28 January 2016

Accepted 25 April 2016

Published Online First 23 May 2016
Thorax 2016;71:950-951.

doi:10.1136/thoraxjnl-2016-208409

\section{REFERENCES}

1 Field JK, Duffy SW, Baldwin DR, et al. UK Lung Cancer RCT Pilot Screening Trial: baseline findings from the screening arm provide evidence for the potential implementation of lung cancer screening. Thorax 2016:71:161-70.

2 Baldwin DR, Duffy SW, Wald NJ, et al. UK Lung Screen (UKLS) nodule management protocol: modelling of a single screen randomised controlled trial of low-dose CT screening for lung cancer. Thorax 2011;66:308-13.

3 Lopes Pegna A, Picozzi G, Mascalchi M, et al. Design, recruitment and baseline results of the ITALUNG trial for lung cancer screening with low-dose CT. Lung Cancer 2009:64:34-40.

4 Lopes Pegna A, Picozzi G, Falaschi F, et al. Four-year results of low-dose CT screening and nodule management in the ITALUNG trial. J Thor Oncol 2013:8:866-75.

5 Das M, Mühlenbruch G, Mahnken AH, et al. Asbestos Surveillance Program Aachen (ASPA): initial results from baseline screening for lung cancer in asbestos-exposed high-risk individuals using low-dose multidetector-row CT. Eur Radiol 2007;17:1193-9.

6 de-Torres JP, Marín JM, Casanova C, et al. Identification of COPD patients at high risk for lung cancer mortality using the COPD-LUCSS-DLCO. Chest 2016;149:936-42.

7 Carozzi FM, Bisanzi S, Falini P, et al. Molecular profile in body fluids in subjects enrolled in a randomised trial for lung cancer screening: perspectives of integrated strategies for early diagnosis. Lung Cancer 2010;68:216-21.

8 Sozzi G, Boeri M, Rossi M, et al. Clinical utility of a plasma-based miRNA signature classifier within computed tomography lung cancer screening: a correlative MILD trial study. J Clin Oncol 2014;32: 768-73.

9 Field JK, van Klaveren R, Pedersen JH, et al. European Randomized Lung Cancer Screening Trials: Post NLST. J Surg Oncol 2013;108:280-6. 